\title{
Nonlinearity-tailored fiber laser technology for low-noise, ultra-wideband tunable femtosecond light generation
}

\author{
Xiaomin Liu, ${ }^{1,2, \dagger}$ Jesper LaegsgaArd, ${ }^{1, \dagger}$ (i) Roman Iegorov, ${ }^{3,4}$ Ask S. Svane, ${ }^{1}$ F. Ömer Ilday, ${ }^{3,5}$ \\ Haohua Tu, ${ }^{6}$ Stephen A. Boppart, ${ }^{6}$ and Dmitry Turchinovich ${ }^{2,7, *}$ \\ ${ }^{1}$ DTU Fotonik, Technical University of Denmark, 2800 Kgs. Lyngby, Denmark \\ ${ }^{2}$ Max Planck Institute for Polymer Research, Ackermannweg 10, 55128 Mainz, Germany \\ ${ }^{3}$ Department of Physics, Bilkent University, 06800 Ankara, Turkey \\ ${ }^{4}$ National Research Tomsk Polytechnic University, Institute of Power Engineering, 30 Lenin Avenue, 634050 Tomsk, Russia \\ ${ }^{5}$ Department of Electrical and Electronics Engineering, Bilkent University, 06800 Ankara, Turkey \\ ${ }^{6}$ Biophotonics Imaging Laboratory, University of Illinois at Urbana-Champaign, Urbana, Illinois 61801, USA \\ ${ }^{7}$ Fakultät für Physik, Universität Duisburg-Essen, Lotharstraße 1, 47048 Duisburg, Germany \\ ${ }^{*}$ Corresponding author: dmitry.turchinovich@uni-due.de
}

Received 31 July 2017; revised 10 October 2017; accepted 26 October 2017; posted 31 October 2017 (Doc. ID 303292); published 27 November 2017

\begin{abstract}
The emission wavelength of a laser is physically predetermined by the gain medium used. Consequently, arbitrary wavelength generation is a fundamental challenge in the science of light. Present solutions include optical parametric generation, requiring complex optical setups and spectrally sliced supercontinuum, taking advantage of a simpler fiber technology: a fixed-wavelength pump laser pulse is converted into a spectrally very broadband output, from which the required resulting wavelength is then optically filtered. Unfortunately, this process is associated with an inherently poor noise figure, which often precludes many realistic applications of such supercontinuum sources. Here, we show that by adding only one passive optical element-a tapered photonic crystal fiber-to a fixed-wavelength femtosecond laser, one can in a very simple manner resonantly convert the laser emission wavelength into an ultra-wide and continuous range of desired wavelengths, with very low inherent noise, and without mechanical realignment of the laser. This is achieved by exploiting the double interplay of nonlinearity and chirp in the laser source and chirp and phase matching in the tapered fiber. As a first demonstration of this simple and inexpensive technology, we present a femtosecond fiber laser continuously tunable across the entire red-green-blue spectral range. (๑) 2017 Chinese Laser Press
\end{abstract}

OCIS codes: (140.3538) Lasers, pulsed; (140.3510) Lasers, fiber; (140.3600) Lasers, tunable; (190.4370) Nonlinear optics, fibers; (060.7140) Ultrafast processes in fibers; (140.7300) Visible lasers.

https://doi.org/10.1364/PRJ.5.000750

\section{INTRODUCTION}

Wavelength-tunable ultrafast light sources, with tunability far beyond the gain bandwidth of (a limited number of) naturally available laser media, enable a great variety of applications ranging from bio-spectroscopy [1-3] to safety inspection [4]. Presently available solutions, such as optical parametric generation (OPG), can provide laser emission extending from the ultraviolet (UV) to the mid-infrared (MIR) [5,6]. However, the need for (intra-cavity) mechanical readjustment for wavelength tuning results in operational reliability issues, limiting the applications of OPG sources outside dedicated optical laboratories. On the other hand, supercontinuum (SC) fiber lasers usually have simple configuration and are operationally robust $[7,8]$. However, for many applications, efficient power conversion in SC technology usually conflicts with the strict requirement on low noise [9], affecting negatively the system stability and precluding acceptably fast measurements.

Fiber-optic Cherenkov radiation (FOCR), also known as dispersive wave generation [10] or non-solitonic radiation [11], is a remarkably efficient process, in which one can convert the wavelength of a standard mode-locked pump laser (e.g., Yb-, Er-, or Tm-fiber laser) to almost any target wavelength. Unlike SC generation, the in-fiber Cherenkov process allows for wavelength conversion in a spectrally isolated, resonant fashion, at modest pump pulse energies, and with low noise [12]. In this process, soliton compression of subpicosecond pump pulses in a fiber with anomalous dispersion 
at the pump wavelength leads to the emission of a dispersive wave, or FOCR, at a wavelength determined by the phase matching to the soliton wavenumber [13]. As illustrated in Fig. 1(a), by varying the photonic crystal fiber (PCF) dispersion profile, and thus the FOCR phase-matching condition by controlling the lateral dimension of a PCF structure, one can easily tune the resulting FOCR wavelength over a broad spectral range. A number of groups have utilized this flexibility to obtain efficient generation of relatively narrowband, spectrally isolated FOCR pulses in the visible and UV ranges [14-22].

Because of its straightforward implementation and good noise figure, FOCR generation has been successfully used in biophotonics, including such demanding applications as biomedical imaging [23]. However, the ultimate challenge of achieving continuous ultra-broadband laser tunability still stands: in all systems demonstrated to date, the FOCR wavelength was fixed to within a few tens of nanometers [22] by the specific dispersion of the fiber and the wavelength of the pump laser. However, for many applications, a much wider tunability is desired, e.g., for fluorescence spectroscopy, one might want a single source spanning the

(a)

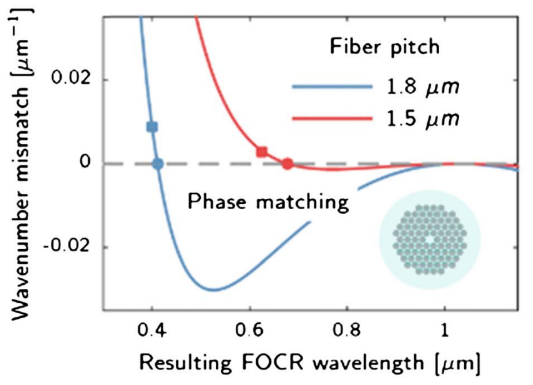

(c)

Continuous FOCR wavelength tunability

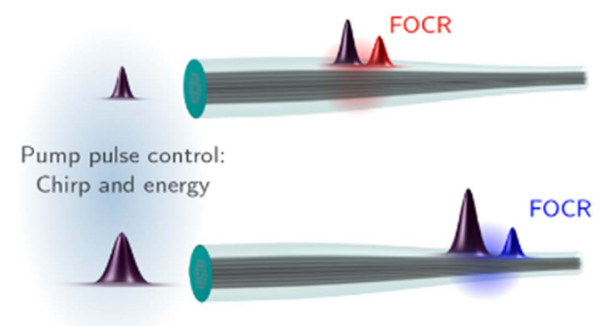

entire visible region. Discrete wavelength switching could be achieved by manually changing between the PCFs with different dispersion profiles $[15,16,19]$, or, equivalently, by switching between the pump lasers with different pump wavelengths [18] — a demanding task for a practical implementation of this technique. True arbitrary wavelength generation-spectrally continuous wide-range laser tunability, was still unattainable [14,24].

In this work, we demonstrate a novel widely tunable femtosecond fiber laser based on FOCR in tapered PCFs pumped by a $\mathrm{Yb}$-based femtosecond laser whose power and pulse duration could be widely tuned. The location of the soliton compression point in the fiber depends on the pump pulse parameters, and the FOCR wavelength depends on the position of said compression point due to the fiber taper. Given that the laser parameters themselves are intricately interrelated [25], it is essential to regard the entire laser-tapered fiber setup as a single strongly coupled nonlinear dynamical system. As will be shown below, our design allows generation of continuously tunable FOCR spanning the wavelength range 414-612 nm using a single nonlinear fiber and a single pump source with a fixed

(b)

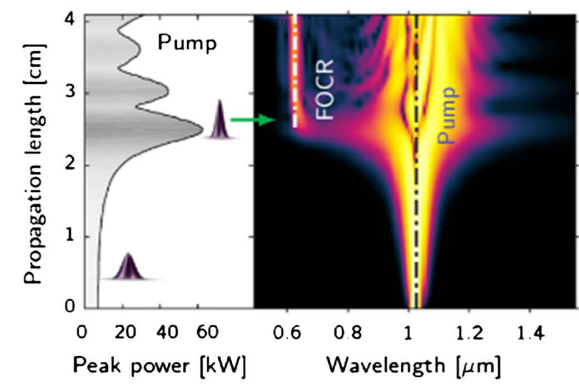

(d)

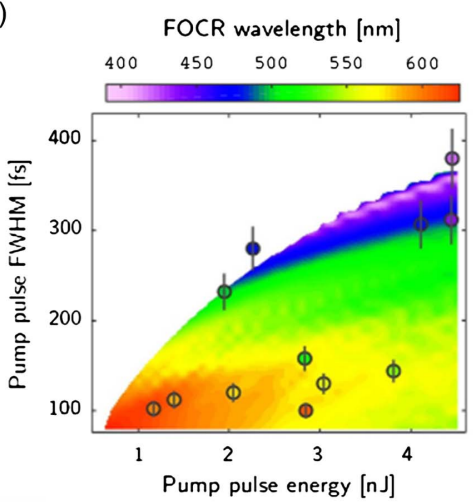

Fig. 1. Principles of continuously tunable femtosecond fiber laser. (a) Phase-matching curves for FOCR generation for different fiber pitch dimensions, allowing for FOCR generation at different wavelengths in the visible range from a pump pulse of $1035 \mathrm{~nm}$ central wavelength. Circles indicate phase-matched FOCR wavelengths in the limit of weak pump power, while squares indicates the phase-matching points for a typical peak power of $100 \mathrm{~kW}$. Inset: representative image of a PCF structure. (b) Peak power evolution of the pump pulse (left) and simulated spectrum (right) along a tapered PCF for FOCR generation at $580 \mathrm{~nm}$ from a transform-limited Gaussian input pump pulse at $1035 \mathrm{~nm}$. FOCR is generated around the point of maximum soliton compression of the pump pulse in the fiber, as indicated by the green arrow. (c) Illustration of continuous FOCR tunability in a tapered PCF by a combination of power and pulse duration control of a fixed-wavelength pump pulse. Such control of the pump pulse determines the point of maximum pump pulse compression, which is the FOCR generation point within the taper, as shown in (a). The FOCR wavelength, in its turn, is defined by the local dispersion of the tapered PCF at this generation point, according to the pump-to-FOCR phase-matching condition such as shown in (a). (d) Average visible wavelength for numerically simulated FOCR spectra, as a function of pump pulse energy and duration. Well-defined FOCR peaks appear close to the FOCR generation threshold, whereas for increasing pulse energy/decreasing duration, continuum formation ensues. Circles are the experimental results. The error bars represent the uncertainty in deconvolution factor of the pulse duration from the measured autocorrelation, as explained in the Appendix A.2. 
central wavelength. The principal idea behind our scheme is applicable to other wavelength ranges and waveguide types, and we believe the technique has considerable potential for improvement with optimized fiber and laser design. In particular, it may allow for very rapid wavelength tuning by purely electric control of the pump source, without any need for mechanical rearrangements.

\section{GENERAL PRINCIPLE OF WIDELY TUNABLE CHERENKOV FIBER LASER}

Our scheme ultimately relies on the following facts. First, FOCR generation requires pump-to-FOCR phase matching, which dictates the resulting FOCR wavelength based on the pump pulse central wavelength and the fiber dispersion, as illustrated in Fig. 1(a). Second, the FOCR generation process is strongly power dependent, and is, hence, spatially localized around the point of maximum soliton compression of the pump pulse in the fiber, as seen from the numerical simulation in Fig. 1(b). In a tapered fiber, with the dispersion profile varying along the fiber length, the resulting FOCR wavelength will thus be determined by the local fiber dispersion properties at this maximum-compression point within the fiber. In its turn, the actual position of this point along the fiber length, and hence the resulting output wavelength of the Cherenkov laser, can be unambiguously and continuously determined simply by varying the duration and power of the input pump pulse, as illustrated in Fig. 1(c).

Figure 1(d) illustrates the parameter space for continuous ultra-wide tuning of the central wavelength of the fiber taper-based Cherenkov laser demonstrated in this work, which is defined by the energy and duration of the pump pulses derived form a mode-locked $\mathrm{Yb}$-fiber laser. As the figure shows, for a given pulse duration there is a threshold energy for FOCR generation. Close to this threshold, low-noise spectrally isolated FOCR peaks appear, whereas a further increase of pump pulse energy leads to continuum formation and increased noise. Since our goal is to achieve low-noise FOCR peaked around a chosen central wavelength, it is clear from the figure that we need to control both pump pulse energy and duration. This is because one needs to achieve a pump pulse energy close to the FOCR generation threshold (which itself depends on the FOCR wavelength), while at the same time ensuring that the soliton compression happens at the right position within the taper. Further details on the calculation and the experiment are described below in the text.

While many variations on the above general principle can be envisioned, in the present paper we focus on the generation of femtosecond pulses continuously tunable over the visible spectrum in the 400-600 $\mathrm{nm}$ range. We chose this spectral range for its special importance for bio-photonics applications, which we foresee as one of the main beneficiaries of our new technology. There, such a wide tuning range will allow the user to easily match the central wavelength of the laser to both one- or twophoton absorption peaks of molecules of interest [26]. As a result, both single-photon $[1,26,27]$ and multiphoton [26-32] microscopy can be conveniently performed with the same laser system and microscope without any setup readjustment.

\section{RESULTS AND DISCUSSION}

\section{A. Experimental Layout}

Our experimental setup is illustrated in Fig. 2 (see Appendix A.3 for details). The pump laser is a standard-design mode-locked femtosecond $\mathrm{Yb}$-fiber master oscillator power amplifier (MOPA) laser operating at $42 \mathrm{MHz}$ repetition rate, with a central wavelength of $1035 \mathrm{~nm}$, maximum pulse energies in excess of $10 \mathrm{~nJ}$, and pulse durations tunable upwards from $90 \mathrm{fs}$ [33]. The pump pulses are easily reshaped in terms of peak power, duration, and chirp by tuning the fiber amplifier and/or the compressor (shadowed box in Fig. 2). The structural profile of the PCF used in both the experiments and the simulations is shown in Appendix A.4.

\section{B. Modeling}

Our numerical simulations are based on a generalized nonlinear Schrödinger equation adapted to tapered fibers [34], parameterized by plane-wave-based calculations of the PCF dispersion and effective area properties. In the simulations, we assume that the fiber structure is preserved through the taper, except for an overall scaling that follows the outer diameter of the fiber, which we can experimentally measure. Furthermore, we consider single-mode propagation of linearly polarized transformlimited Gaussian pulses, whose duration and power are varied. Birefringence may be included, but has been neglected in the presented results, because the birefringence of the PCF used in the experiments is unintentional and not well characterized. To obtain the desired spectrally smooth narrow-bandwidth FOCR pulses, the precise taper shape is found to play an important role. From theory, we expect the accelerated downtaper as shown in Fig. 2 to yield the cleanest FOCR pulse shapes, as explained in Appendix A.5, and such a taper was used in this work.

\section{Ultra-Wide Continuous Laser Tunability}

The upper panel of Fig. 3(a) shows the simulated FOCR spectra by pumping the designed down-tapered PCF with transform-limited Gaussian pulses at $1035 \mathrm{~nm}$, and varying the duration and power of the pump pulses for FOCR tunability.

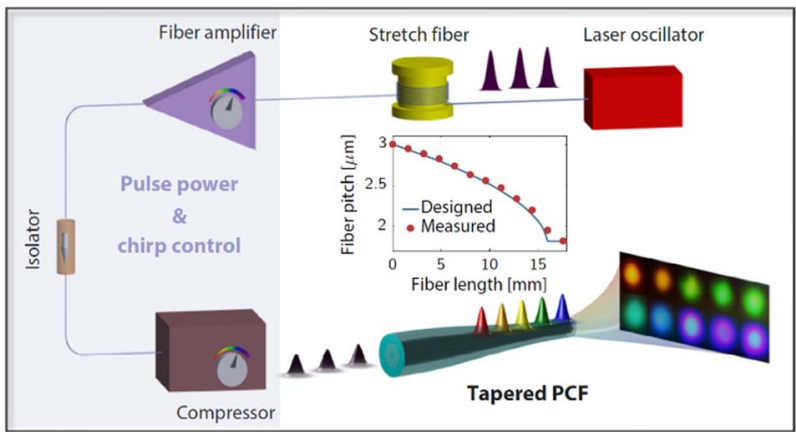

Fig. 2. Experimental setup. Simplified schematic of the tunable femtosecond Cherenkov fiber laser. By adjusting the power and compression settings, or the power alone, of a fixed-wavelength pump pulse provided by a standard mode-locked fiber laser, the output FOCR wavelength from a PCF taper is continuously tuned in a wide spectral range. Inset: the designed (solid line) and its practical realization (dots) of a PCF taper profile. 
(a)

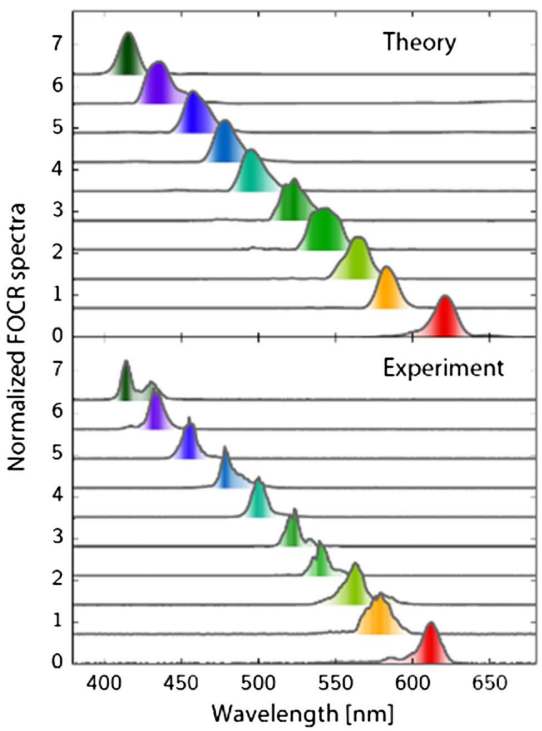

(b)

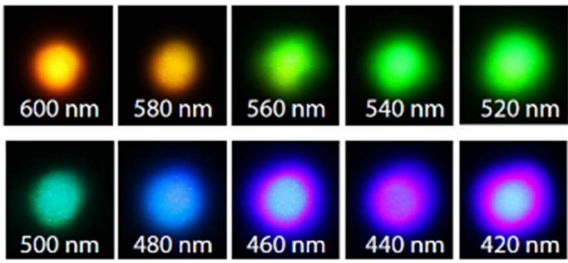

(c)

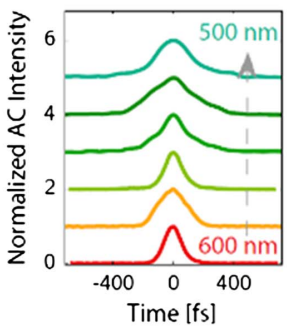

Fig. 3. Characterization of a widely tunable femtosecond fiber laser. (a) Simulated and experimentally measured spectra of tunable FOCR. (b) The far-field images of the output light. (c) Left: the measured autocorrelation curves of FOCR signals. Right: the autocorrelation FWHM calculation (dashed line) and measurement (dots) of the generated FOCR pulses, and of the pump pulses (squares).

The simulation results are also shown in Fig. 1(d), where the parameter space for the resulting laser output tunability-the $1035 \mathrm{~nm}$ pump pulse duration and energy-is presented. The experimentally obtained spectra between 414 and $612 \mathrm{~nm}$ are depicted in the lower panel of Fig. 3(a) and the corresponding experimental conditions (Appendix A.6, the power and duration of the input pump pulse) are shown in Fig. 1(d). As one can see, the broad laser tunability within $\sim 200 \mathrm{~nm}$ spectral range is conveniently achieved by electrical control of the fiber amplifier power and the pulse compressor, with the experimental results closely matching the theoretical predictions.

In the above experiments, the laser output wavelength was tuned in a matter of seconds, by manually adjusting the amplifier gain and the compressor setting (see shadowed box in Fig. 2). Remarkably, even by adjusting the pump power alone, and keeping the compressor setting fixed, we could tune the laser wavelength over the $420-560 \mathrm{~nm}$ range, covering much of the visible spectrum. This constitutes a further simplification of the practical implementation of our technology, drastically speeding up the laser tuning process. Indeed, the electronic control of pump power allows its adjustment on $\sim 10 \mu$ s timescale (Appendix A.7), and can, in principle, be scaled down even to nanoseconds with complementary modulation of the laser signal [35]. Such a capability to achieve fully electrical ultrawideband tunability of a laser on a microsecond, eventually even sub-microsecond timescale, can enable a new class of demanding applications where high laser tuning rate is of essence.

Figure 3(b) shows the far-field mode profiles of the measured FOCR signals, which were observed to be stable, with no sign of higher-order mode admixture, although the PCF used was multimode at visible wavelengths. Figure 3(c), left panel, shows the experimental autocorrelations of the FOCR output, measured in the operational range of our autocorrelator, 500-600 nm. Just as expected from the simulations, we obtain smooth pulse spectral profiles with moderate variations in duration. In Fig. 3(c), right panel, the autocorrelation full width at half-maximum (FWHM) of Cherenkov pulses is shown as a function of their wavelength (blue dots), along with the corresponding values for the input pump pulses from a $\mathrm{Yb}$-fiber laser at $1035 \mathrm{~nm}$ (red squares). For comparison, in the same figure the autocorrelation FWHM of the simulated FOCR pulses is shown (dashed blue line), being corrected for dispersive effects in the optical elements between the PCF and the autocorrelator in order to match the experimental conditions (Appendix A.8). We note that the simulated pulse durations at the fiber end facet were below $100 \mathrm{fs}$ at all FOCR wavelengths, including the spectral range below $500 \mathrm{~nm}$, for which the experimental data could not be obtained due to the limitations of our autocorrelator and related optics. These generated pulses are much shorter than what is typically produced by currently most-used spectrally sliced SC technology [32]. This means that higher pulse peak power can be achieved with comparable pulse energy, benefitting such applications of tunable ultrafast lasers as nonlinear spectroscopy and microscopy.

Our simulations quite accurately reproduce both the measured spectra of the Cherenkov output in the entire range of laser tunability, 414-612 nm [Figs. 1(d) and 3(a)], and the measured autocorrelations within the accessible experimental range above $500 \mathrm{~nm}$, while predicting the output pulse duration with reasonable accuracy [Fig. 3(c), right panel]. This fact strongly supports the presented physical picture of widely tunable FOCR generation in fiber tapers. We note that our simulations are performed using an idealized Gaussian pump laser pulse shape, while in reality the pump pulses may have a more complicated time structure. Nevertheless, even using such a simplification in the modeling, a close agreement is achieved between the experimental data and the theoretical predictions. 
We comment that the actual limits to wavelength tunability of the Cherenkov fiber laser are defined by the interplay between the pump wavelength and soliton peak power, as well as the fiber dispersion at the pump and target wavelengths [12], all of which can be engineered. To this end, the laser wavelength tunability exceeding 1 octave of frequencies using a single taper can be envisaged.

\section{Laser Output Power and Noise Figure}

Figure 4(a) shows the generated FOCR output power, and the pump-to-FOCR power conversion efficiency of our laser system. The output power range $1-5.5 \mathrm{~mW}$ corresponds to pulse energies of $\sim 25-130 \mathrm{pJ}$, easily satisfying the energy requirements for, e.g., nonlinear microscopy experiments [36]. The observed conversion efficiency of our widely tunable FOCR laser of a few percent is quite typical for this wavelengthconversion process $[37,38]$.

The intensity noise of the light source is another key parameter for laser performance evaluation. Figure 4(b) shows the signal-to-noise ratio (SNR) of the output of our laser, which is 1-3 orders of magnitude better than the typical picosecond-pumped supercontinuum (ps-SC) source spectrally sliced to $\sim 10 \mathrm{~nm}$ bandwidth [38]. We note that spectral slicing of our Cherenkov laser to the same $\sim 10 \mathrm{~nm}$ bandwidth around its peak wavelength will reduce its SNR by only an additional factor of 2. Therefore, using the tunable Cherenkov laser, the spectroscopy or imaging data of the same quality can be obtained up to $2-4$ orders of magnitude faster, as compared to the ps-SC source of the same $\sim 10 \mathrm{~nm}$ bandwidth, clearly demonstrating a significant practical advantage of widely tunable Cherenkov laser technology. The details of laser noise characterization are presented in Appendix A.9. With regards to the spectral stability, we did not observe any noticeable spectral changes during the measurement periods typically lasting up to $10 \mathrm{~min}$.

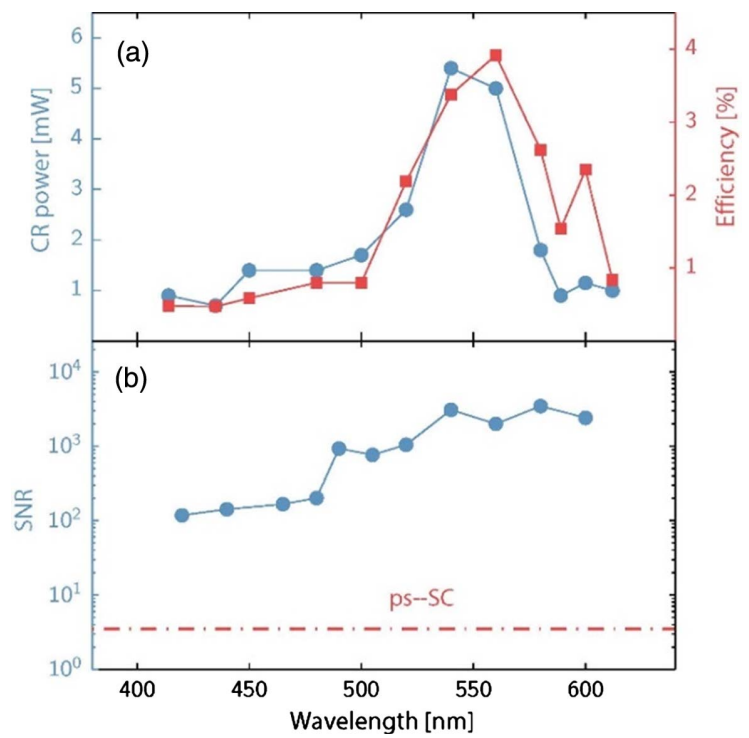

Fig. 4. Output power, conversion efficiency, and noise as a function of laser emission wavelength. (a) Generated FOCR output power (blue dots) and its conversion efficiency (red squares). (b) The SNR of FOCR signals (dots) and of a standard ps-SC source spectrally sliced to $10 \mathrm{~nm}$ bandwidth (FWHM) by optical bandpass filters (dashed line).
Generally, the FOCR noise is found to increase as the wavelength is shortened, which is theoretically expected because the short-wavelength generation requires higher pump soliton numbers, thus translating into higher noise [7]. By the same argument, for a given wavelength the SNR is found to decrease with increasing output FOCR power.

\section{CONCLUSIONS}

We have presented a novel technology for widely tunable and highly stable ultrafast fiber lasers, based on FOCR in fiber tapers. Adding only one passive new element, a fiber taper, to a standard pump pulsed laser allows one in a very simple manner to continuously and resonantly convert the fixed pump laser wavelength to any desired wavelength within an ultra-wide spectral range, in a single-mode output, with power conversion efficiency of a few percent, and with excellent noise characteristics as compared to existing approaches, such as spectrally sliced SC. Based on this idea, we demonstrated a femtosecond fiber laser continuously tunable over the $200 \mathrm{~nm}$ wide redgreen-blue spectral range, emitting 100-200 fs pulses with pulse energies of $\sim 25-130 \mathrm{pJ}$. Our measurements are in good agreement with parameter-free simulations.

Because of its modest requirements on pump pulse energy and duration, monolithic all-fiber integration of a widely tunable Cherenkov laser [22] is a straightforward technological perspective. The demonstrated basic principle of combining tailored PCF taper structures with agile femtosecond pump lasers, such as widely commercially available $\mathrm{Yb}-$, Er-, or Tm-fiber lasers, could be implemented in many ways, to target the generation within different spectral and intensity ranges, as well as discrete wavelengths, multiple pulses, etc. For example, when pumped with a standard Er-fiber laser at $1550 \mathrm{~nm}$, the Cherenkov femtosecond output with higher energy [39] as well as broader tunability could be generated. Our findings thus pave the way to practical applications of simple and inexpensive Cherenkov femtosecond fiber lasers with wide continuous tunability in industry, science, and medicine.

As one of the key applications for the demonstrated laser technology, we foresee bio-photonics, making use of oneand many-photon excitations, Raman processes, etc., in biological tissues as well as in specially added markers (see, e.g., Refs. $[27,40])$. Currently, substantial effort is being put into translating bio-spectroscopy techniques, demonstrated and tested in research laboratories, into clinical environments. Here, the widely tunable Cherenkov fiber lasers will satisfy most stringent requirements on enabling light sources, such as adjustment-free long lifetime operation, compactness and robustness, high operational stability, sufficiently fast wavelength tuning, and very low noise. This will drastically, by several orders of magnitude, minimize the acquisition time of highquality imaging and spectroscopy data, as compared to existing laser technologies [14,24], thus enabling measurements that were unfeasible before. Potentially, a single widely tunable Cherenkov laser will be able to access the whole range of fluorophores $(\sim 400-800 \mathrm{~nm})$ used both in confocal microscopy and superresolution microscopy, thereby providing a complete multimodality laser scanning solution in a translational environment. 


\section{APPENDIX A}

\section{PCF}

The tapered fiber used in this work is based on the small-core nonlinear PCF NL-3.0-850 (NKT Photonics A/S), which has a zero-dispersion wavelength (ZDW) around $850 \mathrm{~nm}$. The definition of fiber pitch $\Lambda$ is shown in Fig. 5.

\section{Error Bars in Fig. 1(d)}

The error bars represent the estimated uncertainty in deconvolution factor of the actual pulse FWHM duration from the measured autocorrelation. As an error we chose the $8.7 \%$ relative difference of the deconvolution factors for a Gaussian pulse $(c=1.414)$, used for the deconvolution of experimental autocorrelations, and another common pulse shape-the sech $^{2}$ pulse $(c=1.545)$.

\section{Experimental Setup}

The experimental setup of the tunable FOCR laser is shown as Fig. 6. The laser system consists of two main parts: a home-made femtosecond $\mathrm{Yb}$-fiber laser with MOPA configuration at $1035 \mathrm{~nm}$ wavelength and $42 \mathrm{MHz}$ repetition rate used as the pump source and a tapered PCF used for tunable FOCR wave conversion. The pump source is comprised of four main stages: a passively mode-locked oscillator using nonlinear polarization rotation, a stretch fiber, a $\mathrm{Yb}$-fiber amplifier, and a grating pair as the pulse compressor. The oscillator setup is similar to the laser described in Ref. [41]. After amplification, the stretched pump pulse signal can be compressed down to $90 \mathrm{fs}$ by a grating pair with the energy up to $50 \mathrm{~nJ}$. We note here that, for the FOCR generation experiments, pump pulse energy only up to $11.2 \mathrm{~nJ}$ is needed. The amplifier configuration and the associated dynamics were originally explored in Ref. [33]. The coupling efficiency from the pump laser into the tapered PCF is around 50\%.

We also note here that due to wavelength limitation of the bandpass filter inside the autocorrelator in our setup, FOCR pulse duration with the FOCR wavelength shorter than $500 \mathrm{~nm}$ could not be measured. The typical spectrum of the pump laser is shown as the inset of Fig. 6.

During the experiments, both the output pump pulse energy and pulse duration were controlled by two parameters: control of the pump signal amplifier power and the grating pair distance.

\section{Tapered PCF Preparation}

As for the tapered PCF, a Vytran LDS1250 splicing workstation is used to prepare all the tapers needed for the experiments.

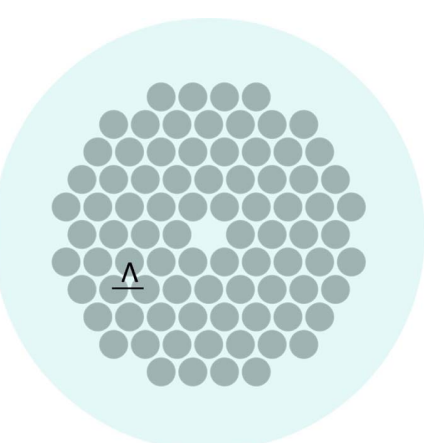

Fig. 5. Representative image of the PCF. $\Lambda$ is the PCF fiber pitch.

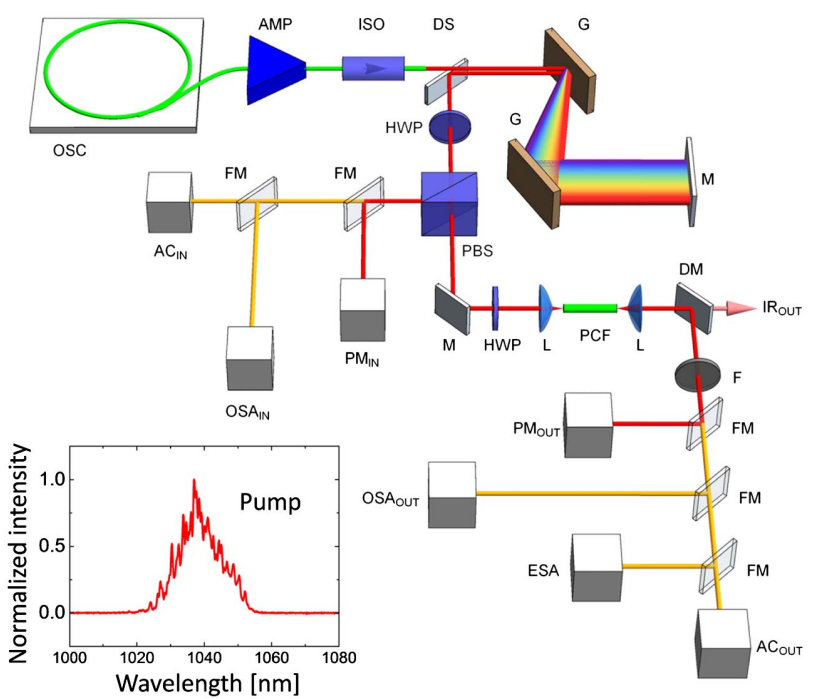

Fig. 6. Experimental setup of the tunable FOCR laser. OSC, pump oscillator; AMP, pump amplifier; ISO, pump isolator; G, grating; M, high reflection mirror; HWP, half-wave plate; PBS, polarization beam splitter; FM, flip mirror; AC, autocorrelator; PM, photometer; OSA, optical spectrum analyzer; DM, dichroic mirror; F, optical bandpass filter; ESA, electrical spectrum analyzer with photodiode. Inset: typical spectrum of the pump laser output.

The taper profiles are then characterized with a microscope. The tapered PCF used both in the experiments and simulations presented in the main text has an initial $25 \mathrm{~mm}$ uniform "lead" section and a following $16 \mathrm{~mm}$ down-tapering part with decreasing diameter. The tapering ratio (taper waist divided by the initial PCF diameter) is 60\%. The taper rate increases toward the waist, as shown in the inset of Fig. 2 in the main text.

Besides the taper mentioned in the main text, some other examples of different designs and their measured taper profiles are shown in Fig. 7. More than 40 linear and 20 non-linear tapers with tapering ratios up to $60 \%$ have been prepared. All of them fit well with the calculated profiles, which demonstrates the high level of tapering accuracy and repeatability.
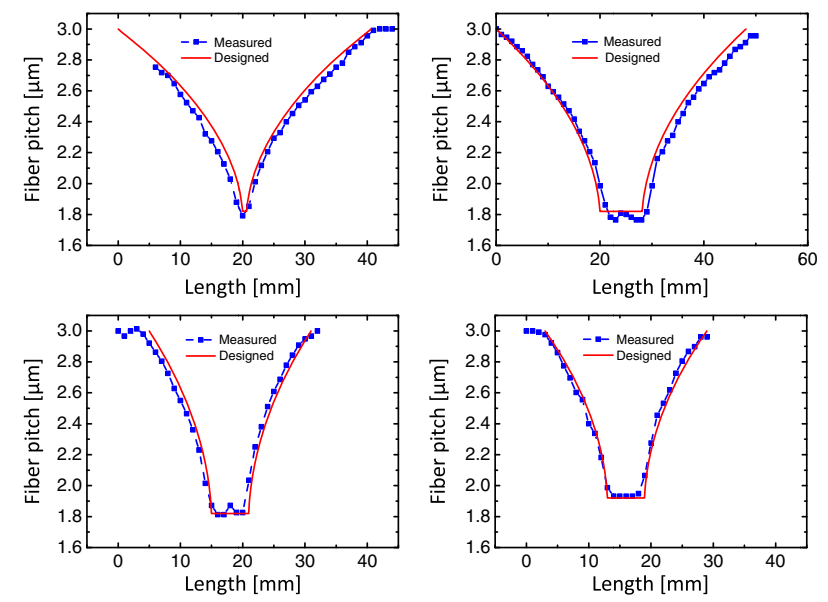

Fig. 7. Examples of different designs (solid line) and their practical realizations (dots) for PCF tapers. 


\section{Theory and Numerical Simulations}

In this work, the simulations of pulse propagation in short tapered PCFs relies on a formulation of $1+1$-dimensional propagation equations for tapered fibers developed in Ref. [34]. The electric field is expanded into a sum of local waveguide modes according to

$$
\begin{aligned}
E(r, t) & =\frac{1}{\sqrt{2 \pi}} \sum_{m} \int \mathrm{d} w A_{m}(z, w) e_{m}\left(r_{\perp}, z, w\right) e^{i\left[w t-B_{m}(w, z)\right]} ; \\
B_{m}(z, w) & =\int_{0}^{z} \mathrm{~d} z^{\prime} \beta_{m}\left(w, z^{\prime}\right),
\end{aligned}
$$

where $\beta_{m}(w, z)$ are the frequency-dependent propagation constants of the different waveguide modes, indexed by $m$. Although the fiber structure under consideration is multimode at both pump and FOCR wavelengths, only propagation in the fundamental mode is considered here. This is justified by the short length of the tapers, and the feasibility of selective coupling into the fundamental mode. In the simulations we also assume propagation in a single linear polarization state, which may not be in full accordance with the experimental situation. However, the PCF is not intentionally birefringent, and although it is likely it has some unintentional birefringence, we cannot estimate this property with an accuracy that would allow for more detailed modeling of polarization properties.

Under these assumptions, the mode index, $m$, in Eq. (A1) may be dropped, and the propagation equation then reads

$$
\begin{gathered}
\frac{\partial A(z, w)}{\partial z}=\quad i \frac{w}{c} n_{2} \sqrt{2 \pi} \int \mathrm{d} w_{1-2}\left[A_{\mathrm{eff}}(z, w)\right]^{-\frac{1}{4}} \tilde{A}\left(z, w_{1}\right) \\
\times \tilde{A}^{*}\left(z, W_{2}\right) \tilde{A}\left(z, w-w_{1}+w_{2}\right) \\
\times e^{i B(w, z)}\left[\left(1-f_{R}\right)+f_{R} R\left(\mathbf{r}, w-w_{1}\right)\right] \\
\tilde{A}(z, w)=\frac{A(z, w) e^{-i B(w, z)}}{\left[A_{\mathrm{eff}}(z, w)\right]^{1 / 4}} .
\end{gathered}
$$

Here, $n_{2}$ is the nonlinear coefficient of silica, and $f_{R}$ is the fraction of the silica nonlinear response function contributed by the delayed (Raman) response, described by the response function $R\left(\mathbf{r}, w-w_{1}\right)$. The necessary input parameters for the model are then the fiber propagation constant $\beta(w)$ and the effective area, $A_{\text {eff }}(z, w)$, as a function of frequency for the fundamental mode. These parameters are determined by using the freely available MIT Photonic-bands software package [42], which solves the Maxwell equations for guided fiber modes by expanding the dielectric function and magnetic field vector in plane waves. This software is used in combination with a perturbative technique for including the effects of material dispersion $[34,43]$, which is crucial in determining the FOCR phase-matching wavelengths.

The pure-silica PCFs are modeled as a cobweb geometry, as illustrated in the inset of Fig. 8. The structural parameters defined in the figure were chosen as $r_{c}=0.2 \Lambda, r_{c c}=0.45 \Lambda$, $w_{b}=0.05 \Lambda, \Lambda=3.2 \mu \mathrm{m}$. With these parameters, experimental results for FOCR generation in untapered fibers were reproduced reasonably well. When describing tapered fibers, it is assumed that the taper simply scales $\Lambda$, while leaving the parameters $\frac{r_{c}}{\Lambda}, \frac{r_{c c}}{\Lambda}$, and $w_{b} / \Lambda$ invariant. The dispersion curves of fibers tapered to various dimensions are displayed in the

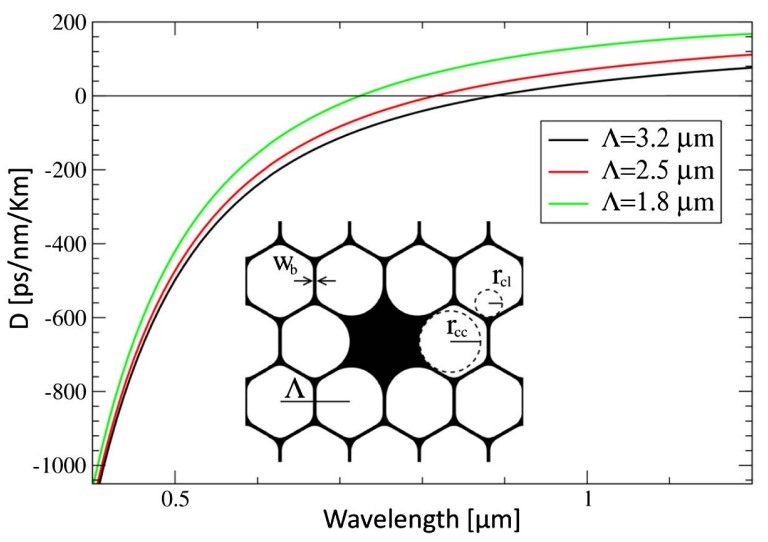

Fig. 8. Dispersion curves for three different $\Lambda$ values of the PCF structure shown in the inset.

main panel of Fig. 8. The tapering leads to an overall increase of the dispersion coefficient over the wavelength range of interest, and shifts the ZDW from 888 to $725 \mathrm{~nm}$.

The Cherenkov phase-matching condition is given by [13]

$\beta(w)-\beta\left(w_{0}\right)-\beta_{1}\left(w_{0}\right)\left(w-w_{0}\right)=\frac{\gamma P_{0}}{2} ; \quad \gamma=\frac{2 \pi n_{2}}{\lambda_{0} A_{\mathrm{eff}}\left(w_{0}\right)}$,

where $w_{0}$ is the pump frequency, $\beta_{1}=\mathrm{d} \beta / \mathrm{d} w$, and $P_{0}$ is the peak power of the pump pulse at the point where the Cherenkov radiation is generated. Typical pump peak powers attained in our simulations are of the order of $100 \mathrm{~kW}$. Figure 1(a) in the main text illustrates how the down-tapering of the PCF allows tuning the FOCR phase-matching point from about $625 \mathrm{~nm}$ down to $400 \mathrm{~nm}$, for $P_{0}=100 \mathrm{~kW}$, by changing the linear dispersion of the fiber. On the other hand, the nonlinear term affects only minor shifts of the phase-matching point, especially at short FOCR wavelengths, where the phase-matching curve is steep.

The phase-matching criterion in Eq. (A4) may be rewritten as

$$
\int_{w_{0}}^{w} \mathrm{~d} w^{\prime}\left[\beta_{1}\left(w^{\prime}\right)-\beta_{1}\left(w_{0}\right)\right]=\frac{\gamma P_{0}}{2} .
$$

Since the pump should be in the anomalous dispersion regime, $D>0$ (otherwise soliton compression will not occur), and $\beta_{1}$ is equal to the inverse group velocity, it follows that the left-hand side (LHS) of Eq. (A5) will initially be negative as $w$ increases from $w_{0}$. This is because, for anomalous dispersion, the group velocity increases with frequency. For the LHS to eventually become positive as $w$ increases, $\beta_{1}(w)-$ $\beta_{1}\left(w_{0}\right)$ must reverse its sign, i.e., the FOCR phase matching will necessarily occur at a frequency where the FOCR has a lower group velocity than the pump. On the other hand, as the PCF is down-tapered, the ZDW moves to higher frequencies, and the group velocity at a given visible frequency (not necessarily the FOCR frequency) increases relative to the pump group velocity. These observations are of crucial importance to the following discussion.

Figure 9(a) and the upper left cartoon of Visualization 1 show an example of a simulated spectral evolution for a linear taper profile. The PCF has a straight lead section of $2.5 \mathrm{~cm}$, with $\Lambda=3.2 \mu \mathrm{m}$, and is then downtapered to $\Lambda=1.92 \mu \mathrm{m}$, 

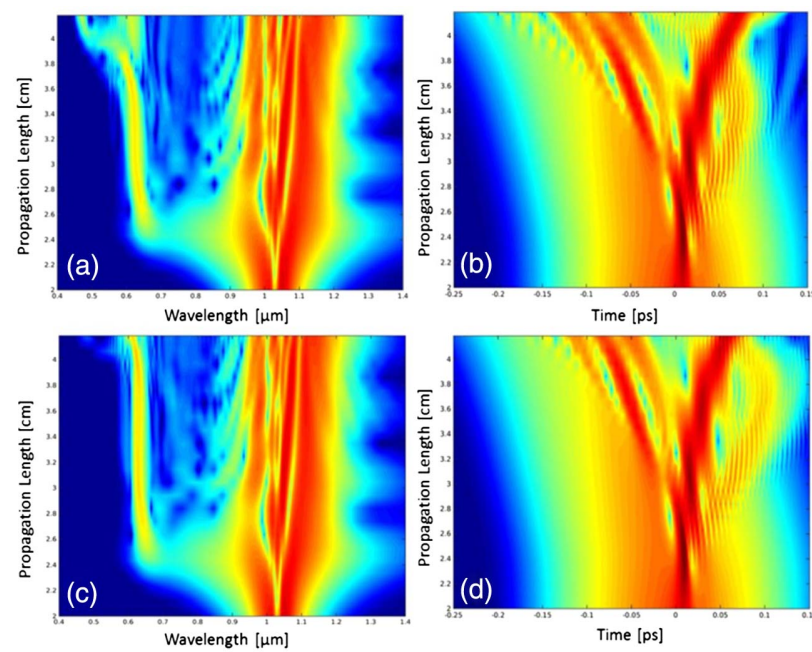

Time [ps]

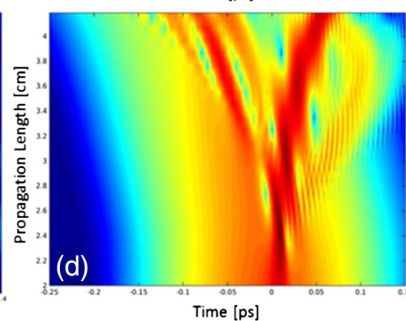

Fig. 9. Simulations of FOCR generation in linear and nonlinear taper profiles. (a), (c) Spectrum and (b), (d) temporal power profile versus propagation distance $z$ in a (a), (b) linear and (c), (d) nonlinear taper profile, with taper and input pulse parameters as described in the text. Both spectral density ( $\mathrm{pJ} / \mathrm{THz}$ ) and power (W) is plotted logarithmically. See also Visualization 1 and Visualization 2 for simulations of FOCR generation in linear and nonlinear taper profiles, respectively.

i.e., to $60 \%$ of its initial diameter, over a distance of $1.6 \mathrm{~cm}$, in which $\Lambda$ varies linearly with distance. A final straight tail section of $0.8 \mathrm{~mm}$ is also included. The input pump pulse has a peak power of $7370 \mathrm{~W}$ and a duration (FWHM) of $105 \mathrm{fs}$, which ensures maximal compression, and FOCR generation, at the end of the lead section, $z=2.5 \mathrm{~cm}$.

The FOCR generation is seen to occur over a distance of $1-2 \mathrm{~mm}$ (note that it is the logarithm of the spectral density that is plotted), with a slight blueshift occurring immediately after the generation point. Hereafter, the FOCR spectrum is relatively constant until around $z=3.8-4 \mathrm{~cm}$, where the spectrum suddenly breaks up with a strongly blueshifted component appearing, having a spectral density comparable to the remaining peak at the original FOCR wavelength. Such a composite spectrum is incompatible with our purpose, which is to generate a single spectral peak whose central wavelength is tunable.

The observed spectral evolution may be rationalized by considering the evolution of the temporal power profile, depicted in Fig. 9(b) and the right two cartoons of Visualization 1. The FOCR initially moves slower than the pump pulse, and is seen to emerge at later times, to the right of the figure. The overlap between pump and visible wavelengths is evidenced by strong interference fringes. The initial blueshift of the FOCR can then be interpreted as a cross-phase modulation (XPM) effect resulting from being on the trailing edge of the stronger pump pulse. As the fiber down-tapers, the relative velocity of pump and FOCR pulses reverses its sign, and the FOCR pulse is seen to catch up with the pump, with a re-collision occurring around $z=3.8 \mathrm{~cm}$. As the FOCR passes through the pump pulse, it is initially blueshifted by XPM, but subsequently redshifts as it emerges on the leading edge of the pump pulse, since XPM spectral shifts are proportional to the time derivative of the pump pulse profile. However, part of the FOCR pulse becomes trapped behind the pump, and is continually blueshifted in the manner described by Skryabin and Gorbach $[44,45]$. As a result of this effect, the blue spectral peak is formed.

A straightforward mitigation scheme for this re-collision effect is to introduce a nonlinear taper profile, as depicted in the inset of Fig. 2. In this taper, the initial taper rate is reduced, so that the FOCR pulse propagates a longer distance with slower velocity than the pump pulse. The taper rate gradually increases, so that the final value of $\Lambda$ is the same as before.

The difference in taper rate from start to end of the tapered section is a factor of 10 . Figures 9(c), 9(d), and the inset plot of Visualization 2 show spectral and temporal evolutions of the same pump pulse as described above in the nonlinear taper. It is clearly seen that the re-collision between pump and FOCR is now delayed until the very end of the taper, and the resulting spectral modifications are much weaker. It should be noted, however, that as the pump power is increased, the emerging pump soliton is increasingly redshifted, and thereby slowed down due to the anomalous dispersion at wavelengths above $1 \mu \mathrm{m}$. Therefore, pump power is ultimately limited by the re-collision effect, even in nonlinear tapers, and the power of the input pulse used to generate the inset plots in Fig. 9 is around the maximal useful power for generation of a clean spectrum above $600 \mathrm{~nm}$ in the nonlinear taper profile.

It should also be noted that the re-collision effect is absent if the FOCR is generated in an up-tapering fiber. This approach suffers from other limitations, which will be discussed in detail elsewhere. However, our main reason for choosing the downtapering profile can be understood from the dispersion curves in Fig. 8. It can be seen that the dispersion coefficient reaches values of around $-1000 \mathrm{ps} / \mathrm{nm} / \mathrm{km}$ at a wavelength of $400 \mathrm{~nm}$, regardless of the value of $\Lambda$. In contrast, the dispersion at $600 \mathrm{~nm}$ is around $-200 \mathrm{ps} / \mathrm{nm} / \mathrm{km}$, and decreases significantly in magnitude with $\Lambda$. If we imagine generating blue FOCR at the beginning of an up-tapering section having a length of $1.6 \mathrm{~cm}$, the dispersive broadening of an FOCR pulse with $10 \mathrm{~nm}$ bandwidth can be estimated to be around $160 \mathrm{fs}$. A similar estimate for a down-tapering profile, where light at $600 \mathrm{~nm}$ is generated at the start of the taper, would predict a broadening of around 30-35 fs. As shown below, we have indeed found that visible FOCR pulses from our down-tapered design emerge from the fiber end facet with FWHM durations below $50 \mathrm{fs}$. In calculations for up-tapering fibers (not shown), we correspondingly find that the short-wavelength pulses are broadened to 100-200 fs, leading to a 1 order-of-magnitude difference between pulse durations at different wavelengths.

To investigate the potential of the proposed FOCR generation scheme, a large number of simulations were performed for the nonlinear taper profile, varying both power and duration of the Gaussian pump pulse, in order to obtain compression and FOCR emission at different points in the taper. A selection of spectra and temporal pulse profiles at the fiber end facet are shown in Fig. 10. The selection criterion was to obtain the highest FOCR energy without compromising spectral isolation, which is evaluated in a purely subjective and nonquantitative way. It can be seen that spectral energy densities around $1.7-2.4 \mathrm{pJ} / \mathrm{nm}$ are obtained for few-nanojoule pump pulses with durations in the 100-300 fs range. As the pump 

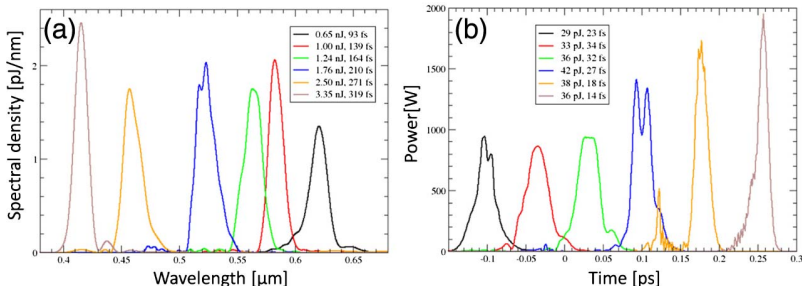

Fig. 10. Simulated tunable FOCR spectral profiles and temporal pulses. Selected (a) spectral profiles and (b) temporal pulses obtained after short-pass filtering the spectrum at $750 \mathrm{~nm}$ as simulated in the nonlinear taper structure when varying pump power and duration. The temporal pulses have been artificially shifted along the time axis for better viewing.

pulse duration is shortened, and/or the power is increased, the compression point moves toward earlier sections of the taper, leading to a longer FOCR wavelength being generated.

The temporal pulses are found to have FWHM durations between 14 and $34 \mathrm{fs}$, with the blue spectra having the shortest duration, as they are generated near the end of the taper, and therefore see little dispersion. Total FOCR energies range between 29 and $42 \mathrm{pJ}$, which in a $42 \mathrm{MHz}$ laser, as was used in the experiments, would correspond to average visible powers between 1.22 and $1.76 \mathrm{~mW}$.

\section{Experimental Conditions}

Table 1 shows the generated FOCR wavelength, as a function of pump pulse energy and duration, as shown in Fig. 1(d). We note here that Gaussian pulse shape was assumed for the pump pulse duration measurements. We also note that, in our system for the FOCR wavelength tunability, only the pump pulse energy and duration are the key parameters. The chirp of the pulses matters only when the pump spectral bandwidth is much broader, which is not the case in our work.

\section{Response Time of Electronic Control of Pump Power}

As shown in Fig. 11, electronic control of pump power allows adjustment of power in few-microseconds timescale, which is limited by the driving electronics. In Ref. [35], we have characterized the response to pump modulation for the output

Table 1. Experimental Conditions of the Pump Pulses as Shown in Fig. 1(d)

\begin{tabular}{lcc}
\hline $\begin{array}{l}\text { Central } \\
\text { Wave [nm] }\end{array}$ & Pump Energy [n]] & Pump Pulse FWHM [fs] \\
\hline 414 & 4.45 & 380 \\
435 & 4.44 & 312 \\
450 & 5.67 & 320 \\
480 & 2.26 & 280 \\
500 & 1.95 & 232 \\
520 & 2.83 & 158 \\
540 & 3.81 & 144 \\
560 & 3.03 & 130 \\
580 & 2.05 & 120 \\
589 & 1.39 & 112 \\
600 & 1.16 & 102 \\
613 & 2.84 & 100 \\
\hline
\end{tabular}

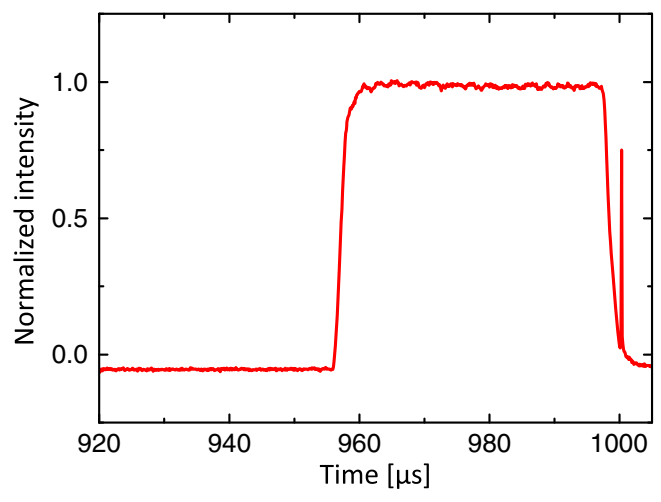

Fig. 11. Optical pump power response profile dependent on the electronic control of pump power.

power of a fiber amplifier, including the same type of gain fiber and diode laser as the pump as used in this work. As to be expected, the transfer of any modulation of the pump laser is limited in frequency due to the finite response of the gain medium. These results show that about a 10\% modulation of the signal power is possible upon full modulation of the pump laser at frequencies $>100 \mathrm{kHz}$. Therefore, a $10 \mu$ s response time is relatively easy to achieve with straightforward modulation of the laser. This level of modulation is achieved easily using simple, low-cost driver electronics. Even this is not a fundamental limitation, since it was demonstrated in the same reference that, just as the transfer of the modulation of the pump rolls of at high frequencies, transfer of modulation of the signal increases concurrently. This leads to the possibility of complementary modulation of both the pump and signal of an ultrafast fiber amplifier without any evident frequency limitation. Modulation of the signal was obtained in that study using an acousto-optic modulator, which can also be implemented with an electro-optic modulator. Therefore, should the need arise, there is no laser-physics-based limitation to how fast the output power of an ultrafast fiber amplifier system can modulate. It would be limited in practice by the electronics, but we routinely achieve rise or fall times of $<10 \mathrm{~ns}$ in other work [38]. Since the propagation of each pulse in the amplifier/ compressor is physically independent of the others, the future possibility of tuning our FOCR wavelength at a timescale of nanoseconds is evident.

\section{Pulse Duration}

From the experimental setup of Fig. 6, we can see that a focusing lens ( $1.9 \mathrm{~mm}$ thick), a bandpass filter $(2 \mathrm{~mm}$ thick, filtering out the residual pump signal), and a half-wave plate $(0.312 \mathrm{~mm}$ thick) together with one $\mathrm{BBO}$ crystal $(0.3 \mathrm{~mm}$ thick $)$ as part of the autocorrelator are used between the laser output and the autocorrelator for the FOCR pulse duration measurements. Therefore, the measured autocorrelation FWHM of FOCR pulses shown in Fig. 2(c) also include pulse chirp induced by the dispersion of the optical components mentioned above. To compare the simulated FOCR pulse duration with the experimental measurements, the autocorrelation FWHM of the simulated FOCR pulses shown in Fig. 2(c) are carefully corrected for dispersive effects in the optical elements between the PCF 
Table 2. Simulated Pulse Duration and Autocorrelation Corrected for Dispersive Effects in the Optical Elements Between the PCF and the Autocorrelator

\begin{tabular}{|c|c|c|c|c|c|c|c|c|}
\hline $\begin{array}{l}\text { Central } \\
\text { Wave } \\
{[\mathrm{nm}]}\end{array}$ & $\begin{array}{l}\text { Simulated } \\
\text { Pulse } \\
\text { Duration } \\
{[\mathrm{fs}]}\end{array}$ & $\begin{array}{c}\text { Dispersion } \\
\text { of } \\
\text { Focus Lens } \\
\text { [fs/nm] }\end{array}$ & $\begin{array}{c}\text { Dispersion } \\
\text { of } \\
\text { Filter } \\
\text { [fs/nm] }\end{array}$ & $\begin{array}{c}\text { Dispersion } \\
\text { of } \\
\text { Half-Wave } \\
\text { Plate [fs/nm] }\end{array}$ & $\begin{array}{c}\text { Dispersion } \\
\text { of } \\
\text { BBO } \\
{[\mathbf{f s} / \mathbf{n m}]}\end{array}$ & $\begin{array}{c}\text { Pulse Duration } \\
\text { After Optical } \\
\text { Elements } \\
{[\mathrm{fs}]}\end{array}$ & $\begin{array}{c}\text { Calculated } \\
\text { Total } \\
\text { Autocorrelation } \\
\text { FWHM [fs] }\end{array}$ & $\begin{array}{c}\text { Measured } \\
\text { Autocorrelation } \\
\text { FWHM } \\
\text { [fs] }\end{array}$ \\
\hline 620 & 22.7 & -1.63 & -0.52 & -0.08 & -0.16 & 55.6 & 91.7 & 126 \\
\hline 583 & 34.1 & -2.02 & -0.64 & -0.1 & -0.19 & 64.05 & 92.6 & 206 \\
\hline 564 & 32 & -2.27 & -0.72 & -0.11 & -0.22 & 102.4 & 136.7 & 133 \\
\hline 495 & 23.5 & -3.65 & -1.11 & -0.17 & -0.34 & 87.3 & 149.1 & 229 \\
\hline 478 & 22.7 & -4.17 & -1.25 & -0.2 & -0.39 & 95.3 & 160.4 & - \\
\hline 457 & 18.5 & -4.96 & -1.46 & -0.23 & -0.45 & 101.2 & 164.5 & - \\
\hline 436 & 14.8 & -5.98 & -1.71 & -0.27 & -0.54 & 149.4 & 224.5 & - \\
\hline 416 & 14.3 & -7.26 & -2.01 & -0.31 & -0.64 & 99.6 & 197.3 & - \\
\hline
\end{tabular}
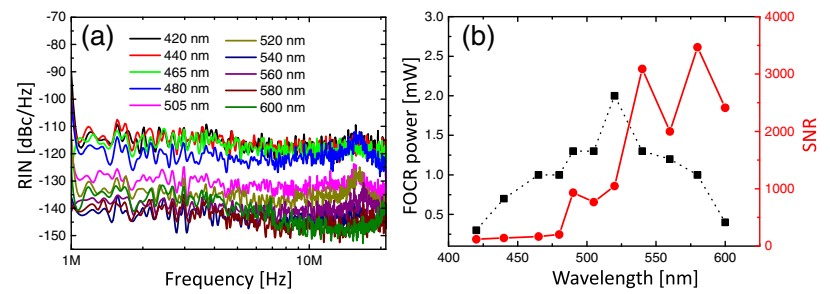

Fig. 12. Noise measurements of the FOCR pulses dependent on the output wavelength. (a) The RIN spectra of the FOCR pulses versus the FOCR wavelength. (b) The SNR of FOCR dependent on the wavelength (red circles) and the corresponding output power (black squares).

and the autocorrelator. The details are shown in Table 2. The right two columns of Table 2 show the corrected autocorrelation FWHM of the simulated FOCR pulses and the measured autocorrelation FWHM separately, as plotted in Fig. 3(c).

\section{Intensity Noise Measurements}

The details of the laser noise measurements can be found in Refs. $[38,46]$. The noise spectra were measured in the baseband of the electrical frequency ranging from 1 to $21 \mathrm{MHz}$ (half of the repetition rate of the pump laser source).

Figure 12(a) shows the measured noise spectra of the output pulses dependent on the FOCR wavelength, and Fig. 12(b) shows the calculated SNR as well as the corresponding output power.

Figure 13(a) shows the optical spectra of the typical FOCR output from the laser system as measured with and without the bandpass optical filters. The FWHM bandwidth of the filters is $10 \mathrm{~nm}$. The power of un-filtered FOCR in this case is $1.3 \mathrm{~mW}$. The noise spectra of the filtered and un-filtered FOCR signals are shown in Fig. 13(b). Figure 13(c) shows the optical intensity spectra of the spectrally unfiltered FOCR, and the SNR spectra measured from the bandpass-filtered FOCR signals such as shown in Fig. 13(a). The SNR of the spectrally unfiltered FOCR is calculated to be 931, while the SNR of the filtered FOCR with the central wavelength at $490 \mathrm{~nm}$ is
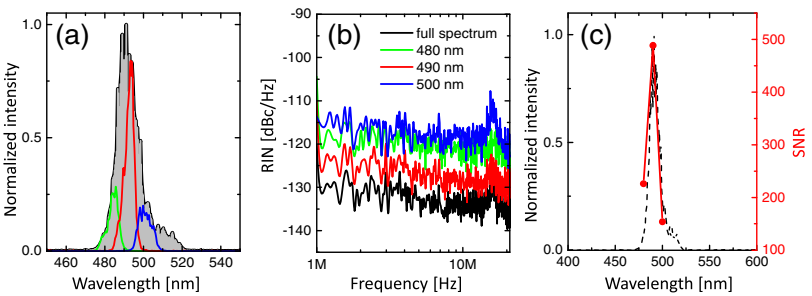

Fig. 13. Noise measurements of the FOCR pulses after bandpass optical filters. (a) FOCR spectra with and without bandpass optical filters. The power of unfiltered FOCR is $1.3 \mathrm{~mW}$. (b) The RIN spectra of FOCR measured with and without bandpass optical filters. (c) Spectrum of FOCR output (black dashed line) and SNR measured with bandpass filters at different spectral positions (red circles). The SNR of the spectrally unfiltered FOCR is 931.
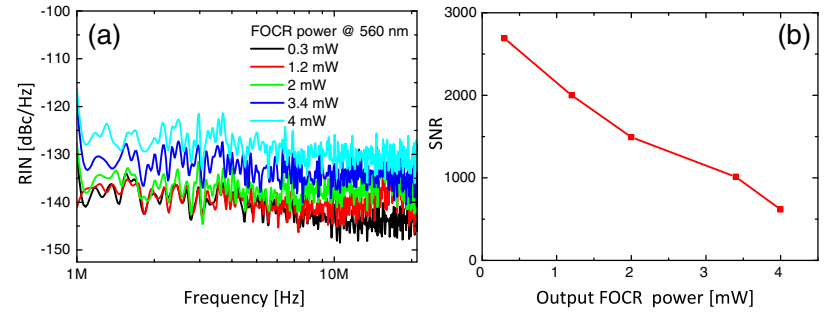

Fig. 14. Noise measurements of the FOCR pulses dependent on the output power. (a) Noise spectra of the FOCR pulses versus output power at FOCR wavelength of $560 \mathrm{~nm}$. (b) The SNR of FOCR dependent on the output power.

488.4. Therefore, as it is written in the main text, our measurements indicate that a filtering of the FOCR spectrum reduces the SNR by only a factor of 2 for a filter centered on the FOCR peak.

Figure 14(a) shows the relative intensity noise (RIN) spectra of the FOCR signal dependent on the output power at FOCR wavelength at $560 \mathrm{~nm}$, while the corresponding SNR is shown in Fig. 14(b). As written in the main text, the SNR decreases as the output power increases. 
Funding. Teknologi og Produktion, Det Frie Forskningsråd (FTP, DFF) (ALFIE); Research Executive Agency (REA) (EU Career Integration Grant 334324 LIGHTER); H2020 European Research Council (ERC) (ERC-617521 NLL); National Cancer Institute (NCI) (1 R01 CA166309); Max-Planck-Gesellschaft (MPG).

Acknowledgment. The authors thank Ö. Akçaalan for taper cleaving, U. Møller, P. Elahi, and H. Kalaycığlu for providing equipment for the experiments, and I. Pavlov, M. Bonn, M. Grechko, and S. Parekh for valuable discussions.

${ }^{\dagger}$ These authors contributed equally to this work.

\section{REFERENCES}

1. G. McConnell, "Confocal laser scanning fluorescence microscopy with a visible continuum source," Opt. Express 12, 2844-2850 (2004).

2. H. Song, S. B. Cho, D. U. Kim, S. Jeong, and D. Y. Kim, "Ultrahigh-speed phase-sensitive optical coherence reflectometer with a stretched pulse supercontinuum source," Appl. Opt. 50, 4000-4004 (2011).

3. J. He, J. Miyazaki, N. Wang, H. Tsurui, and T. Kobayashi, "Biological imaging with nonlinear photothermal microscopy using a compact supercontinuum fiber laser source," Opt. Express 23, 9762-9771 (2015).

4. M. H. Madsen, P. Hansen, M. Zalkovskij, M. Karamehmedović, and J. Garnæs, "Fast characterization of moving samples with nano-textured surfaces," Optica 2, 301-306 (2015).

5. M. H. Dunn and M. Ebrahimzadeh, "Parametric generation of tunable light from continuous-wave to femtosecond pulses," Science 286, 1513-1517 (1999)

6. N. Savage, "Optical parametric oscillators," Nat. Photonics 4, 124-125 (2010).

7. J. M. Dudley, G. Genty, and S. Coen, "Supercontinuum generation in photonic crystal fiber," Rev. Mod. Phys. 78, 1135-1184 (2006).

8. J. M. Dudley and J. R. Taylor, Supercontinuum Generation in Optical Fibers (Cambridge University, 2010).

9. K. L. Corwin, N. R. Newbury, J. M. Dudley, S. Coen, S. A. Diddams, K. Weber, and R. S. Windeler, "Fundamental noise limitations to supercontinuum generation in microstructure fiber," Phys. Rev. Lett. 90, 113904 (2003).

10. G. P. Agrawal, Nonlinear Fiber Optics, 4th ed. (Academic, 2007).

11. J. N. Elgin, T. Brabec, and S. M. J. Kelly, "A perturbative theory of soliton propagation in the presence of third order dispersion," Opt. Commun 114, 321-328 (1995).

12. X. Liu, A. S. Svane, J. Lægsgaard, H. Tu, S. A. Boppart, and D. Turchinovich, "Progress in Cherenkov femtosecond fiber lasers," J. Phys. D Appl. Phys. 49, 23001 (2016).

13. N. Akhmediev and M. Karlsson, "Cherenkov radiation emitted by solitons in optical fibers," Phys. Rev. A 51, 2602-2607 (1995).

14. F. W. Wise, "Femtosecond fiber lasers based on dissipative processes for nonlinear microscopy," J. Sel. Top. Quantum Electron. 18, 1412-1421 (2012).

15. F. Lu, Y. Deng, and W. H. Knox, "Generation of broadband femtosecond visible pulses in dispersion-micromanaged holey fibers," Opt. Lett. 30, 1566-1568 (2005)

16. F. Lu and W. H. Knox, "Generation, characterization, and application of broadband coherent femtosecond visible pulses in dispersion micromanaged holey fibers," J. Opt. Soc. Am. B 23, 1221-1227 (2006).

17. S. P. Stark, J. C. Travers, and P. St. J. Russell, "Extreme supercontinuum generation to the deep UV," Opt. Lett. 37, 770-772 (2012).

18. H. Tu and S. A. Boppart, "Ultraviolet-visible non-supercontinuum ultrafast source enabled by switching single silicon strand-like photonic crystal fibers," Opt. Express 17, 17983-17988 (2009).
19. G. Chang, L.-J. Chen, and F. X. Kärtner, "Highly efficient Cherenkov radiation in photonic crystal fibers for broadband visible wavelength generation," Opt. Lett. 35, 2361-2363 (2010).

20. N. Y. Joly, J. Nold, W. Chang, P. Hölzer, A. Nazarkin, G. K. L. Wong, F. Biancalana, and P. St.J. Russell, "Bright spatially coherent wavelength-tunable deep-UV laser source using an Ar-filled photonic crystal fiber," Phys. Rev. Lett. 106, 203901 (2011).

21. P. St. J. Russell, P. Hölzer, W. Chang, A. Abdolvand, and J. C. Travers, "Hollow-core photonic crystal fibres for gas-based nonlinear optics," Nat. Photonics 8, 278-286 (2014).

22. X. Liu, J. Lægsgaard, U. Møller, H. Tu, S. A. Boppart, and D. Turchinovich, "All-fiber femtosecond Cherenkov radiation source," Opt. Lett. 37, 2769-2771 (2012).

23. M.-C. Chan, C.-H. Lien, J.-Y. Lu, and B.-H. Lyu, "High power NIR fiberoptic femtosecond Cherenkov radiation and its application on nonlinear light microscopy," Opt. Express 22, 9498-9507 (2014).

24. C. Xu and F. W. Wise, "Recent advances in fibre lasers for nonlinear microscopy," Nat. Photonics 7, 875-882 (2013).

25. D. Turchinovich, X. Liu, and J. Laegsgaard, "Monolithic all-PM femtosecond Yb-fiber laser stabilized with a narrow-band fiber Bragg grating and pulse-compressed in a hollow-core photonic crystal fiber," Opt. Express 16, 14004-14014 (2008).

26. M. Yamanaka, K. Saito, N. I. Smith, Y. Arai, K. Uegaki, Y. Yonemaru, K. Mochizuki, S. Kawata, T. Nagai, and K. Fujita, "Visible-wavelength two-photon excitation microscopy for fluorescent protein imaging," J. Biomed. Opt. 20, 101202 (2015).

27. M. Drobizhev, N. S. Makarov, S. E. Tillo, T. E. Hughes, and A. Rebane, "Two-photon absorption properties of fluorescent proteins," Nat. Methods 8, 393-399 (2011).

28. M. Lippitz, W. Erker, H. Decker, K. E. van Holde, and T. Basché, "Twophoton excitation microscopy of tryptophan-containing proteins," Proc. Natl. Acad. Sci. USA 99, 2772-2777 (2002).

29. C. Li, C. Pitsillides, J. M. Runnels, D. Côté, and C. P. Lin, "Multiphoton microscopy of live tissues with ultraviolet autofluorescence," IEEE J. Sel. Top. Quantum Electron. 16, 516-523 (2010).

30. C. Li, R. K. Pastila, C. Pitsillides, J. M. Runnels, M. Puoris'haag, D. Côté, and C. P. Lin, "Imaging leukocyte trafficking in vivo with twophoton-excited endogenous tryptophan fluorescence," Opt. Express 18, 988-999 (2010).

31. D. Li, W. Zheng, Y. Zeng, Y. Luo, and J. Y. Qu, "Two-photon excited hemoglobin fluorescence provides contrast mechanism for labelfree imaging of microvasculature in vivo," Opt. Lett. 36, 834-836 (2011).

32. W. Zheng, D. Li, Y. Zeng, Y. Luo, and J. Y. Qu, "Two-photon excited hemoglobin fluorescence," Biomed. Opt. Express 2, 71-79 (2011).

33. F. O. Ilday, H. Lim, J. R. Buckley, and F. W. Wise, "Practical all-fiber source of high-power, 120-fs pulses at $1 \mu \mathrm{m}$," Opt. Lett. 28, 13621364 (2003)

34. J. Lægsgaard, "Modeling of nonlinear propagation in fiber tapers," J. Opt. Soc. Am. B 29, 3183-3191 (2012).

35. K. Gürel, P. Elahi, L. Budunoğlu, C. Șenel, P. Paltani, and F. Ö. Ilday, "Prediction of pulse-to-pulse intensity fluctuation characteristics of high power ultrafast fiber amplifiers," Appl. Phys. Lett. 105, 11111 (2014).

36. D. Li, W. Zheng, and J. Y. Qu, "Two-photon autofluorescence microscopy of multicolor excitation," Opt. Lett. 34, 202-204 (2009).

37. H. Tu and S. A. Boppart, "Optical frequency up-conversion by supercontintinuum-free widely-tunable fiber-optic Cherenkov radiation," Opt. Express 17, 9858-9872 (2009).

38. X. Liu, G. E. Villanueva, J. Lægsgaard, U. Møller, H. Tu, S. A. Boppart, and D. Turchinovich, "Low-noise operation of all-fiber femtosecond Cherenkov laser," IEEE Photon. Technol. Lett. 25, 892-895 (2013).

39. H. Tu, J. Lægsgaard, R. Zhang, S. Tong, Y. Liu, and S. A. Boppart, "Bright broadband coherent fiber sources emitting strongly blueshifted resonant dispersive wave pulses," Opt. Express 21, 2318823196 (2013).

40. H. Tu, Y. Liu, D. Turchinovich, M. Marjanovic, J. K. Lyngsø, J. Lægsgaard, E. J. Chaney, Y. Zhao, S. You, W. L. Wilson, B. Xu, M. Dantus, and S. A. Boppart, "Stain-free histopathology by programmable supercontinuum pulses," Nat. Photonics 10, 534-540 (2016). 
41. F. Ilday, J. Chen, and F. Kärtner, "Generation of sub-100-fs pulses at up to $200 \mathrm{MHz}$ repetition rate from a passively mode-locked $\mathrm{Yb}$ doped fiber laser," Opt. Express 13, 2716-2721 (2005).

42. S. G. Johnson and J. D. Joannopoulos, "Block-iterative frequencydomain methods for Maxwell's equations in a planewave basis," Opt. Express 8, 173-190 (2001).

43. J. Lægsgaard, A. Bjarklev, and S. E. B. Libori, "Chromatic dispersion in photonic crystal fibers: fast and accurate scheme for calculation," J. Opt. Soc. Am. B 20, 443-448 (2003).
44. A. V. Gorbach and D. V. Skryabin, "Light trapping in gravity-like potentials and expansion of supercontinuum spectra in photonic-crystal fibres," Nat. Photonics 1, 653-657 (2007).

45. A. V. Gorbach and D. V. Skryabin, "Theory of radiation trapping by the accelerating solitons in optical fibers," Phys. Rev. A 76, 1-10 (2007).

46. R. P. Scott, C. Langrock, and B. H. Kolner, "High-dynamic-range laser amplitude and phase noise measurement techniques," IEEE J. Sel. Top. Quantum Electron. 7, 641-655 (2001). 